\title{
Deprescribing and managing polypharmacy in frail older people: a patient-centred approach in the real world
}

\author{
Lelly Oboh, Mohammed Salman Qadir
}

Department of Pharmacy, Guys and St Thomas NHS Foundation Trust, London, UK

Correspondence to Lelly Oboh, Department of Pharmacy, Guys and St Thomas NHS Trust, Gracefield Gardens, London SW16 2ST, UK; lelly.oboh@nhs.net

Received 2 June 2016 Accepted 28 July 2016 Published Online First 19 August 2016

EAHP Statement 4: Clinical Pharmacy Services
CrossMark

To cite: Oboh L, Qadir MS. Eur J Hosp Pharm 2017:24:58-62.

\section{ABSTRACT}

Polypharmacy is common in people with multiple longterm conditions (LTC) to relieve symptoms and improve quality of life. However, it is also associated with poor outcomes and increased risk of adverse drug events in older people. Older people are seldom involved in therapeutic research, and when the results are applied to those with multiple LTCs, it can increase pill burden and adverse events without necessarily replicating the expected positive outcomes. This article describes a pharmacist-led, patient-centred approach to deprescribing in a 73-year-old diabetic man taking multiple medication, with gastrointestinal (GI) and pain symptoms as well as poor adherence to medicines. The approach considered his perspective and experience of taking his many medicines into account while using the best available research evidence and the clinician's experience. After six visits over 8 weeks, the patient was more involved with self-managing his diabetes, his pain and Gl symptoms improved and overall pill burden was reduced.

\section{BACKGROUND}

There is no universally accepted or validated definition of deprescribing, however majority of the existing definitions describe it as a process or approach to drug reduction, withdrawal or cessation. ${ }^{1}$ Although some definitions mention involving the patient in the process, ${ }^{2}$ not much is available with regard to the most effective way to implement deprescribing in practice. ${ }^{3}$

Barnett et $a l^{3}$ described a patient-centred approach to deprescribing in practice, as part of an overall strategy to manage polypharmacy and optimise medicines' use. It involves (1) assessing patient's needs, (2) defining context and overall goals, (3) identifying all potentially inappropriate medicines (using a validated tool such as STOPP/ START $_{\text {criteria }}{ }^{4}$ ) from an accurate list of medication, (4) assessing risks and benefits in the patient context and discussing with the patient to identify the actual inappropriate drugs and priorities to review, (5) agreeing actions to stop drug or reduce dose, (6) communicating with other relevant parties as appropriate and (7) monitoring, reviewing and adjusting.

This case report is an example of how the patient-centred approach was embedded into a routine medication review by an integrated care clinical pharmacist (ICP) to optimise medicines' use. The ICP is an innovative role based in the community to support frail older people identified as having a medicines-related need to get the best outcomes from taking medicines. They work within integrated teams for older people and receive referrals from community nurses, general practitioners (GPs), geriatricians and other practitioners. The ICP coordinates all aspects of medicines-related care for the patient by addressing polypharmacy, non-adherence and using a coaching approach to empower patients. Within the multidisciplinary teams, they lead the medicines agenda by enabling practitioners to optimise the use of medicines within their scope of practice by providing education, expert advice and support.

The report demonstrates how the ICP engaged with and focused on the patient's needs and experience rather than on the drugs. They sought to identify the various health and psychosocial factors that impacted on medicines' use. Where possible, the patient was given the opportunity to be involved as much as or as little as they wanted.

The medicines-related risks and benefits were prioritised based on the importance to the patient, immediate safety concerns of the ICP as well as best research evidence available. ${ }^{5}$ The ICP employed evidence-based tools to identify potentially inappropriate drugs. By engaging with the patient, the ICP identified drugs that had ceased to provide tangible therapeutic benefits, were adversely affecting functionality and quality of life or at best doing nothing, yet increasing pill burden. A care plan to stop, reduce or change medicines was jointly agreed with the patient. This was subsequently discussed and agreed with the patient's GP outlining who, when and how it will be implemented. Monitoring and follow-up after the review ensured that the changes led to the desired outcomes and minimised the risks of adverse events.

The case study demonstrates the importance of having a lead clinician ${ }^{6}$ to coordinate the care plan and communicate with the patient and others to ensure that the agreed actions are followed.

\section{CASE PRESENTATION \\ Presenting features and symptoms}

This patient is a 73-year-old man who was referred to the ICP by a community matron because of concerns about his medicines. He was struggling to manage his medicines and did not take some of them. The matron had explained the importance of taking his medicines and subsequently organised a 'blister pack' (multicompartment compliance aid) to improve his adherence, but neither intervention was successful.

He also had difficulties managing his diabetes, and his HbA1c fluctuated. He had recently been 
changed from administering his insulin using a syringe and phial to the prefilled pen due to concerns about his poor vision.

The patient presented with chronic pain, feeling dizzy and had fallen a few times recently. He attributed the adverse effects that he experienced to some of his medicines and said this was partly responsible for his non-adherence.

\author{
Medical history \\ - Type 2 diabetic (insulin-dependent) \\ - Right eye diabetic retinopathy \\ - Diabetic neuropathy \\ - Erectile dysfunction \\ - Hypertension \\ - Incontinence \\ - History of urinary tract infection \\ - Falls \\ - Cataracts \\ - Chronic obstructive pulmonary disease (queried) \\ - Memory impairment
}

His estimated glomerular filtration rate was $55 \mathrm{~mL} / \mathrm{min} / 1.73 \mathrm{~m}^{2}$ and body mass index was $22.53 \mathrm{~kg} / \mathrm{m}^{2}$, but both results over a year old. His most recent blood pressure (BP) was 110/ $62 \mathrm{~mm} \mathrm{Hg}$. His latest HbA1c result was 7.9\%, but he was unable to provide information on recent blood glucose levels. His mini mental state examination was $16 / 30$, and he admitted to forgetting to attend his memory clinic appointment for example.

His alcohol consumption was 28 units/week. He smoked 20 cigarettes a day, had tried varenicline a few years ago, but stopped taking due to side effects.

\section{Social history}

The patient lived alone on the third floor of an apartment block. He had no input from social services, but had a private carer who provided support two to three times a week with meal preparation and cleaning. The patient was mobile and often out and about.

\section{Current medication (GP records)}

1. Humulin I KwikPen 100 units/mL_every morning

2. Metformin $500 \mathrm{mg}$ tablets - 2 twice a day (BD)

3. Vardenafil $20 \mathrm{mg}$ tablets-as directed when required

4. Oxybutynin $2.5 \mathrm{mg}$ tablets-1 BD

5. Gabapentin $100 \mathrm{mg}$ caps 1 -three times a day

6. Paracetamol $500 \mathrm{mg}$ tablets-2 when required

7. Movelat Gel-when required

8. Quinine sulfate $200 \mathrm{mg}$ tablets-1 at night

9. Lansoprazole $30 \mathrm{mg}$ caps-1 once a day

10. Amlodipine $10 \mathrm{mg}$ - every morning

11. Ramipril $5 \mathrm{mg}$ caps-1 BD

12. Simvastatin $40 \mathrm{mg}$ tablets-1 at night

13. Salbutamol $100 \mathrm{mcg}$ chlorofluorocarbon (CFC)-free inhaler-when required

14. Seretide 125 CFC-free inhaler-1-2 puffs BD

15. Tiotropium $18 \mathrm{mcg}$ caps for inhalation-once a day

16. Unistik 3 Comfort Lancet (28G $1.8 \mathrm{~mm}$ )

17. Contour Next blood glucose testing strips

18. BGStar glucose testing strips

19. OneTouch Ultrasoft Lancets

\section{Pharmacist actions}

The ICP visited the patient to undertake an in-depth assessment of his medicines-related needs. First, a reconciliation of medicines was performed using the recent hospital discharge summary list, GP brief summary and the patient's own medication present in the home. Then, the ICP used a locally developed tool designed to identify and assess unmet medicines' needs in older people relating to access, adherence and therapeutics. ${ }^{7}$ In line with evidence-based practice, ${ }^{2}$ the ICP considered for each problem, the patient's views, current research evidence and their own expertise. Then, the needs were prioritised, the options discussed and a care plan agreed with the patient. The pharmacist took responsibility to ensure that relevant healthcare professionals received clear communication about the actions to be taken and when. A copy of the care plan was documented in the patient's record.

\section{INVESTIGATIONS}

His seated BP was $110 / 62$, and standing BP after $1 \mathrm{~min}$ $(115 / 59 \mathrm{~mm} \mathrm{Hg})$ was measured to assess for postural hypotension (defined as a difference of $20 \mathrm{~mm} \mathrm{Hg}$ systolic or $10 \mathrm{~mm} \mathrm{Hg}$ diastolic), because it is common in older people and diabetics, and given the patient's history of dizziness and falls. eGFR was requested as there was no record of one in the preceding 12 months. This was needed in view of the patient's history of type 2 diabetes mellitus and because some medicines like ramipril, metformin and gabapentin require close monitoring of renal function for safe dosing and frequency.

\section{MEDICATION REVIEW ACTIONS}

\section{Access to medicines}

At the time, the patient received his medicines dispensed in a multicompartment compliance aid (MCA) 'blister-pack', which he collected every 2 weeks from his community pharmacy. The pharmacy staff ordered and collected his prescriptions on his behalf, but only for the items in the MCA. As a result, he ran out of medicines on some occasions and often had difficulty getting his insulin and consumables. He was unclear whether this was down to him forgetting to send in a repeat request to the GP or to collect the medicines from the pharmacy. The patient was reluctant to agree to have his medicines regularly delivered to him at home, as he was often out. However, he decided that when he needed help, for example, in the winter, he could ask for a delivery service.

He was unable to use the lancing device to draw blood; so, the matron gave him a supply of Unistik 3 Comfort Lancets, but he had run out and was unsure of how to get further supply.

The ICP discussed and agreed with his community pharmacist to put a robust system in place as part of repeat ordering and collection service to check the need for insulin and consumables at the same time the MCA medicines are ordered.

At follow-up, systems had been put in place, and there were no further problems with supply.

\section{Adherence}

Generally, the patient was adherent with his morning doses, omitted the afternoon doses and took some of his evening doses. He was recently changed from Humulin I phials to Humulin I KwikPen due to concerns about his poor dexterity, and also because large air bubbles were observed in the syringe when he tried to draw up the insulin from the phial. He could not see the air bubbles because of his poor vision, which led to variable dosing. The patient had gone back to using his old phial and syringe, because he was unable to remember how to use the KwikPen device.

Also, previously, the patient used a Contour machine and testing strips that had been changed to BGStar machine and strips by his GP. He was unsure of how to use them and as a result was not checking his blood glucose levels prior to insulin administration. 
The ICP showed the patient how to use the KwikPen and visited him daily for 3 days to assess his ability. He still required prompts at various stages and was not comfortable with using the device. The ICP discussed the option of a referral to the district nursing team to teach him or for daily visits to administer insulin. This was not acceptable to the patient as he was not willing to wait indoors for the district nurse to arrive every morning.

As the patient's community pharmacy was $<2$ min walk from his house and he often passed by on his way out, the ICP suggested that the community pharmacist observe and prompt where necessary with his injection technique. The community pharmacist agreed, and the patient was prepared to visit the pharmacy in the morning to demonstrate his insulin administration technique. After five visits over a 2-week period, the patient was confident in using his KwiKpen. The ICP also taught the patient how to use BGStar machine, and he agreed to take the machine on a few occasions to demonstrate how he was using the machine in front of his community pharmacist.

\section{Metformin and gastrointestinal symptoms}

The patient was prescribed 15 medicines (excluding appliances) and a total of 19 doses per day (excluding topicals, inhalers and 'when required' doses). He had a general complaint about taking too many medicines and felt some were not working for him. Specifically, he felt metformin contributed to his gastrointestinal (GI) symptoms, which is why he needed lansoprazole. To remedy this, he reported that he took one instead of two metformin tablets two times per day to minimise his symptoms. However, on closer examination of his MCA, the ICP found that he was not always taking the evening metformin tablets, because he could not tell the difference between amlodipine and metformin tablets.

The ICP explained the importance of taking a suitable dose of metformin regularly and suggested a change to metformin $1 \mathrm{~g}$ modified release formulation once daily to reduce the risk of GI effects ${ }^{8}$ and also reduce pill burden. Metformin is a rational choice in the patient, and his last HbA1c was fairly controlled at $7.9 \%(62.8 \mathrm{mmol} / \mathrm{mol})$ in spite of the non-adherence. Targets can be relaxed in frail older people, those with dementia or at risk of falling. ${ }^{79}$ His latest eGFR was $63 \mathrm{~mL} / \mathrm{min} / 1.73 \mathrm{~m}^{2}$, and so metformin dose was continued with close monitoring of eGFR. The 6-monthly HbA1c trend $(7.4 \%(57.4 \mathrm{mmol} / \mathrm{mol})$, $6.9 \% \quad(51.9 \mathrm{mmol} / \mathrm{mol}), \quad 7.3 \% \quad(56.3 \mathrm{mmol} / \mathrm{mol}), \quad 6.7 \%$ (49.7 mmol $/ \mathrm{mol}), 7.9 \%(62.8 \mathrm{mmol} / \mathrm{mol}))$ was checked to establish an overall picture of stability over a period of time. A change of $0.5 \%$ or more is considered a clinically significant change in diabetic control and may indicate that the patient's non-adherence had an impact on his blood glucose levels.

The patient had been taking lansoprazole for a number of years since he had Helicobacter pylori eradication treatment and found it beneficial for his dyspepsia. Although he did not take it regularly,- he was reluctant to stop or reduce the dose.

Following the change to meformin MR tablets and dose reduction, his GI symptoms improved; so, the patient agreed to reduce lansoprazole to $15 \mathrm{mg}$ daily. It was agreed that this would be dispensed in standard containers, so that he could take two $15 \mathrm{mg}$ capsules if he felt his symptoms worsen. At 8 weeks, he was taking lansoprazole $15 \mathrm{mg}$ three to four times a week.

\section{Oxybutynin}

The patient could not recall experiencing urinary symptoms recently. Oxybutynin has strong anticholinergic properties, which can cause falls and worsen cognitive impairment in older people. Furthermore the immediate release formulation should not be prescribed in older people like the patient who are more predisposed to these adverse effects. ${ }^{10}$

The patient and the GP were in agreement with the ICP suggestion to stop the oxybutynin and monitor.

It was agreed that if the patient experienced bothersome symptoms, then another medication would be trialled.

\section{Analgesia}

The patient's description of the pain in his legs was neuropathic. He seemed to think gabapentin was working well even though he omitted the midday dose due to being out. The patient also took two paracetamol tablets regularly every day for the pain and applied Movelat Gel to his legs, but he did not find either helpful; so, they were stopped.

His gabapentin dose was suboptimal for neuropathic pain, which should be initiated at $300 \mathrm{mg}$ OD and increased accordingly to between 600 and $1800 \mathrm{mg}$ daily in three divided doses, if eGFR is $50-80 \mathrm{~mL} / \mathrm{min} / 1.73 \mathrm{~m}^{2}$. The patient felt his current dose was sufficient, and he did not want to increase his tablet burden; so, the dose was changed to $100 \mathrm{mg} \mathrm{BD}$ to reflect what he was taking.

The patient was taking quinine prn for occasional leg cramps, which he felt was beneficial. He had not taken any for a few months and was regularly disposing off it after taking out of the MCA. As the patient was not experiencing regular nocturnal leg cramps, the ICP suggested stopping the quinine. The patient was agreeable, but he wanted to keep some at home, just in case. So, the quinine was dispensed in its original box, and use monitored over the next few weeks.

The ICP considered the possibility that the leg cramps could be an adverse effect from the interaction of simvastatin and amlodipine. However, it was difficult to ascertain for sure, because the patient did not always take the simvastatin tablets and did not associate it with the cramps. The maximum dose of simvastatin in combination with amlodipine should be $20 \mathrm{mg} .{ }^{11}$

National Institute for Health and Care Excellence recommends high-intensity statins for diabetics; the lower dose was appropriate for the patient in view of his leg cramps. However, simvastatin $40 \mathrm{mg}$ at night was changed to atorvastatin $20 \mathrm{mg}$ in the morning, to facilitate adherence.

\section{BP, falls and dizziness}

The patient had experienced falls and dizziness for which he was receiving strength and balance exercises from the physiotherapist. The falls clinic letter indicated that his falls were more mechanical in nature due to the cluttered flat, and his alcohol consumption may have contributed.

Orthostatic hypotension is more common in diabetes, and an $\mathrm{HbA} 1 \mathrm{c}$ below $7 \%(53.0 \mathrm{mmol} / \mathrm{mol})$ can increase the risk of falls, especially in those taking insulin. ${ }^{8}$

Deciding on the optimal BP target in older people is controversial, and guidance ranges from $140 / 90$ to $150 / 90 \mathrm{~mm} \mathrm{Hg}$ in older diabetics. ${ }^{78}$ However, a recent study showed that a BP of $165 / 85 \mathrm{~mm} \mathrm{Hg}$ in patients over 75 years was associated with lower mortality. ${ }^{12}$ Ramipril and amlodipine are rational drug choices for the patient, considering his comorbidities and nonadherence. There is no difference in BP-lowering effect between once and twice daily ramipril dosing. ${ }^{13}$ Since the patient was not taking the evening dose of ramipril, it was agreed to stop the evening dose. Amlodipine was reduced to $5 \mathrm{mg}$, as the patient had presented with swollen ankles in the past. The ICP monitored his BP on subsequent visits, and there was no significant increase in BP. 


\section{Respiratory}

The patient had an expired Seretide $125 \mathrm{mcg}$ inhaler, and his tiotropium inhalation capsules did not have the HandiHaler device. He was not taking either of them as he was not experiencing any breathlessness, cough or wheezing. His $\mathrm{FEV}_{1}$ was $75 \%$ in 2009 , and $\mathrm{FEV}_{1} / \mathrm{FVC}=0.65$. He mentioned that he was diagnosed with asthma many years ago, but he had not experienced any respiratory symptoms that prevented him from his activities of daily living, and therefore did not want any further investigations. He used salbutamol inhaler when he had a cold or was feeling tired after or on exertion, and this seemed to control his symptoms.

\section{Vardenafil}

The patient reported that he was taking four tablets every 2 months, and he linked metformin to his erectile dysfunction (ED). The ICP reassured him that metformin was not the likely cause and explained that ED is a common complication of type 2 diabetes.

\section{OUTCOME AND FOLLOW-UP}

Following the review, the ICP visited the patient six times over 8 weeks to ensure that deprescribing was done safely. At the end of that period, the patient was more involved with selfadministering his insulin and monitoring his blood glucose without compromising his much desired independence. His pain control and GI symptoms had improved. He knew he was able to go into his local community pharmacy if he had further problems with the supply of his medicines.

The overall number of medicines was reduced from 15 to 10 (excluding appliances), and dosing frequency reduced from 15 to 7 (excluding prn medicines).

\section{DISCUSSION}

Systematic reviews have looked into various interventions to manage polypharmacy in older people, many of which were undertaken by pharmacists in hospital, care home or clinical settings. ${ }^{14} 15$ Although they have demonstrated improvement in surrogate markers of appropriate prescribing, they are yet to prove their clinical significance or impact on patient-related outcomes. In addition, Spinewine et al concluded that interventions undertaken by skilled pharmacists working within a multidisciplinary team produced better results. ${ }^{14}$ Generally, the elements of the interventions in the publications include a review of the medicines and actions taken by healthcare professionals. However, there is little discussion about patient-centredness as described in this case study, for example, taking into account patient choices, the impact of the home environment, social circumstances and functionality.

There were key issues identified in recent literature, ${ }^{16} 17$ about managing polypharmacy in a patient-centred way, that were encountered in the process of deprescribing for the patient. The patient's views and understanding about his medicines and conditions affected his adherence and the extent to which his medicines were optimised. Poor vision, cognitive impairment, reduced ability to learn new tasks, all impacted on his ability to monitor blood glucose, inject insulin and obtain regular supplies of his medicines. Although he was offered help from district nursing service and local pharmacists, he was not always willing to compromise his independence, lifestyle and daily routine. He complained about taking too many medicines and generally was willing for medicines to be stopped, but, when faced with the reality, he was hesitant in a few instances. ${ }^{18}$ Good communication, including exploring his anxieties and negotiating for a trial period, with the assurance that drugs will be monitored and restarted if need be, facilitated safe withdrawal.

Consideration was given to the patient's abilities, impairments and communication styles in order to engage with, educate and empower him to make informed decisions in the process. Established teaching techniques such as repetition, linking to familiar things, hands-on experience, demonstrations, feedback and sequenced visits to build on information proved valuable. In agreeing the action plan, a balance had to be struck between the clinician's therapeutic goals and quality of life from the patient's perspective.

In a similar case study about managing polypharmacy in an older patient with complex needs by Steinman et al, ${ }^{19}$ a physician used a stepwise process. He considered the multiple factors that affected medicines' use from a patient's, carer's and clinician's perspective, as well as rationalisation of drug therapy using research evidence. Specific interventions to resolve the needs identified were also agreed in collaboration with the patient and their carer. Changes to drug therapy were implemented slowly and sequentially over time and monitored closely for benefits and adverse effects.

In the authors' experience, the monitoring and follow-up stage is usually the most time-consuming aspect of the process. Furthermore, the patient's case required more than the average number of visits, and this reflects the complexities of the interventions needed.

Although the ICP is an independent prescriber, they did not prescribe for this patient. However, it is recognised that where there are changes to medicines, an ICP prescriber can facilitate prompt implementation of the action plan and improve outcomes.

\section{Learning points}

- The patient's perspective and involvement in the deprescribing process is important for drugs to be withdrawn safely and outcomes realised.

- Best available research is important for rationalisation of drug therapy.

- Pharmacists working within multidisciplinary teams can play an important role as a lead clinician to coordinate medicines-related care and collaborate with the patients and others involved.

- Deprescribing goes beyond identifying a list of medicines to be stopped, and there should be sufficient time built into the process to allow adequate monitoring and follow-up.

- The role of local community pharmacists providing ongoing support to stable frail older people is worth exploring to build capacity.

Competing interests None declared.

Patient consent Obtained.

Provenance and peer review Not commissioned; externally peer reviewed.

\section{REFERENCES}

1 Reeve E, Gnjidic D, Long J, et al. A systematic review of the emerging definition of 'deprescribing' with network analysis: implications for future research and clinical practice. Br J Clin Pharmacol 2015;80:1254-68.

2 Woodward M. Deprescribing: achieving better health outcomes for older people through reducing medications. J Pharm Pract Res 2003;33:323-8. 


\section{Case report}

3 Barnett NL, Oboh L, Smith K. Patient-centred management of polypharmacy: a process for practice. Eur J Hosp Pharm 2016;23:113-17.

4 O'Mahony D, O'Sullivan D, Byrne S, et al. STOPP/START criteria for potentially inappropriate prescribing in older people: version 2. Age Ageing 2015;44: 213-18.

5 Sackett DL, Straus SE, Richardson WS, et al. Evidence-based medicine: how to practice and teach EBM. 2nd edn. New York: Churchill Livingstone, 2000.

6 Duerden M, Avery T, Payne R. Polypharmacy and medicines optimisation: making it safe and sound. King's Fund Report, 2013. http://www.kingsfund.org.uk/ publications/polypharmacy-and-medicines-optimisation

7 Rosenbloom EK, Goldstein FR. The development of trigger questions to support the case finding of people with unmet medicines-management needs. IJPP 2007;15:21.

8 National Institute of Health and Clinical Excellence. NICE guidelines [NG28]. Type 2 diabetes in adults: management. 2015.

9 International Diabetes Federation. Guideline for managing older people with type 2 diabetes. 2013

10 National Institute of Health and Clinical Excellence. NICE Clinical Knowledge Summary. LUTs in men. 2015.

11 MHRA Drug Safety. Update October 2012. https://www.gov.uk/drug-safety-update/ simvastatin-dose-limitations-with-concomitant-amlodipine-or-diltiazem
12 Ogliari G, Westendorp RG, Muller M, et al. Blood pressure and 10-year mortality risk in the Milan Geriatrics 75+ Cohort Study: role of functional and cognitive status. Age Ageing 2015;44:932-7.

13 Goyal D, Chong AY, Watson RL, et al. Assessment of single versus twice daily dosing of ramipril by ambulatory blood pressure monitoring in patients similar to those included in the HOPE study. J Hum Hypertens 2007;21:525-30.

14 Cooper JA, Cadogan CA, Patterson SM, et al. Interventions to improve the appropriate use of polypharmacy in older people: a Cochrane systematic review. BMJ Open 2015;5:e009235.

15 Spinewine A, Fialová D, Byrne S. The Role of the Pharmacist in Optimizing Pharmacotherapy in Older People. Drugs Aging 2012;29:495-510.

16 Scott IA, Hilmer SN, Reeve E, et al. Reducing inappropriate polypharmacy: the process of deprescribing. JAMA Intern Med 2015;175:827-34.

17 Garfinkel D, Mangin D. Feasibility study of a systematic approach for discontinuation of multiple medications in older adults: addressing polypharmacy. Arch Intern Med 2010;170:1648-54.

18 Reeve E, Wiese MD, Hendrix I, et al. People's attitudes, beliefs, and experiences regarding polypharmacy and willingness to deprescribe. J Am Geriatr SoC 2013;61:1508-14.

19 Steinman MA, Hanlon JT. Managing medications in clinically complex elders: 'There's got to be a happy medium'. JAMA 2010;304:1592-601. 